\title{
Le dépistage systématique des tumeurs digestives
}

Le diagnostic précoce des cancers gastriques et rectaux, de loin les plus fréquemment rencontres dans le tube digestif, est relativement facile en clientele ou à l'hôpital, avec les moyens dont on dispose actuellement; mais leur dépistage méthodique dans les collectivités est un problème infiniment plus délicat, auquel Guy Albot et ses colla-borateurs ont apporté depuis plus de dix ans une solution, dont ils ont relate récemment (Acadêmíe de Médecíne, 17 février 1953:

Bulletin, vol. 137, p. 107) les résultats.

$14^{*}$

182 Editorial

II suffit d'imaginer ce que seraient des examens radiologiques ou endoscopiques massifs de toute une population pour les declarer irréalisables!

Les méthodes sélectives de pré-dépístage consistent en:

Radioscopie gastrique; mais celle-ci laisse échapper presque à coup sûr le cancer au debut. Analyse du sediment du liquide retire de Гestomac, procédé long, coûteux et peu sûr.

Examen détaillé des sujets hypochlorhydriques ou anémiques; mais Гabsence de ces deux anomalies ne permet pas d'exclure le cancer.

C'est ainsi que le choix des auteurs parisiens s'est porté sur le pré-dépístage cliníque à plusieurs echelons: il s'agit de sélectionner les suspects, qui seront seuls pris en charge par le centre de dépistage. Dans une premiere étape, on élimine tous les sujets qui ne présentent aucun trouble digestif. La seconde étape comporte l'exclusion des malades porteurs d'une affection «évidemment bénigne» et des fonctionnels. Ce sont les «organismes naissants de la médecine sociale» (médecins d'entreprises, contrôleurs des assurances, etc.) qui s'oc-cupent de ces deux premiers tours. Le centre de dépistage est done charge, soit des suspects de cancer, soit des malades pour lesquels un diagnostic precis n'a pas été obtenu. Le travail de ce centre doit être confié à des gastro-entérologistes éprouvés, dont un assistant qui decide des examens à faire et élimine les cas simples (lesions mani-festement malignes), tandis que les cas douteux sont reserves à l'appréciation du chef de consultation; encore faut-il que l'expert, ses collaborateurs et son radiologue forment une équipe homogène, condition indispensable pour éviter des erreurs, dans un domaine délicat entre tous.

Au cours des 7 dernières années, le centre dirigé par G. Albot a dépisté 57 cancers sur 2799 malades expertises. Malgré ses apparences modestes, cette proportion est considerable: un cancer méconnu pour 50 malades examines, soit $20,3^{\circ} / 00$, alors que les chiffres les plus favorables publiés ailleurs ne dépassent pas $3 \% 00$. C'est au dépistage par echelon - au lieu d'etre systématique et semblable pour tous qu'il faut attribuer ce succès. M.D. 